\title{
Window length selection for optimum slowness resolution of the local-slant-stack transform
}

\author{
Sergi Ventosa ${ }^{1}$, Carine Simon ${ }^{2}$, and Martin Schimmel ${ }^{3}$
}

\begin{abstract}
One of the most critical decisions in the design of a localslant-stack transform (LSST) is the selection of its aperture, or more precisely, the selection of the appropriate number of traces and their weighting coefficients for each slant stack. The challenge is to achieve a good compromise between the slowness and the spatial resolution. Conventionally, the window size is chosen in a more intuitive manner by visual inspection and some limited tests. We analyzed the LSST to establish rigorous criteria for the window selection to achieve the optimum slowness and spatial resolution in the transformed domain for a given data set. For this purpose, we estimated the slowness resolution in the LSST domain as a function of the spatial-window
\end{abstract}

\begin{abstract}
bandwidth and of the spectral characteristics of the waves. For a wave with a given bandpass spectrum, the slowness resolution, the stopband attenuation, and the wavefront-tracking capability are determined by the spatial window. For narrowband signals, the spatial window must be larger than the stopband bandwidth divided by the desired slowness resolution and the central frequency of the band. For wideband signals, the window length is determined by the lowest frequency components. Much longer windows can only be used when the slowness and the amplitude variations of the wavefront trajectories are small. We validated our approach with a synthetic example and applied it to a wideangle seismic profile to show the filter performance on real data in which the LSST-window length is determined in an automatic, data-adaptive manner.
\end{abstract}

\section{INTRODUCTION}

Several seismic-processing techniques, such as noise filtering and velocity analysis, rely on the fact that seismic measurements are laterally coherent. Interfering, nonstationary noise will hinder these algorithms. Two commonly used features along seismic profiles are: the similarity along signal trajectories, which correspond to wavefronts, and the wave-propagation direction given by the velocity/slowness vector at the receiver. When trace density is high, we employ this high similarity/coherence to design filters depending on apparent slowness — inplane component of the slowness vector - to increase the signal-to-noise $(\mathrm{S} / \mathrm{N})$ ratio.

Mainstream filters for highly coherent signals are based on planewave decomposition techniques. These techniques can be classified into several broad categories: (1) pie-slice $f-k$ (frequency-wavenumber) filters (see, e.g., Embree et al., 1963; Yilmaz and Doherty,
2001); (2) filters in the $\tau$ - $p$ (intercept-time, slope, or slowness) domain, also called linear Radon or slant-stack domains (Stoffa et al., 1981; Durrani and Bisset, 1984; Turner, 1990; Deans, 1993; Yilmaz and Doherty, 2001; Wilson and Guitton, 2007); (3) filters in the slowness-frequency $(p-f)$ domain (Forbriger, 2003; Dev and McMechan, 2009); (4) filters based on eigenvalue decompositions (see, e.g., Vrabie et al., 2004, 2006); (5) filters in the frequency-offset $(f-x)$ domain (see, e.g., Bekara and van der Baan, 2009); and (6), time-space prediction-error filtering (see, e.g., Guitton, 2005). Note the close relation among the first filters; the $f-k$ transform and the slant-stack transform (SST) are related by the projection-slice theorem (e.g., Durrani and Bisset, 1984); and the $p$ - $f$ transform and the SST, by the Fourier transform.

The slowness variations of the signals strongly constrain the level of sparsity that plane-wave-decomposition techniques can achieve. To overcome this limitation, these techniques adopt the following

\footnotetext{
Manuscript received by the Editor 7 October 2010; revised manuscript received 12 August 2011; published online 17 February 2012; corrected version published online 17 February 2012.

${ }^{1}$ IFP Energies Nouvelles, Rueil-Malmaison, France. E-mail: ventosa@ipgp.fr.

${ }^{2}$ Consejo Superior de Investigaciones Científicas (CSIC), Marine Technology Unit, Barcelona, Spain. E-mail: csimon@utm.csic.es.

${ }^{3}$ Consejo Superior de Investigaciones Científicas (CSIC), Institute of Earth Sciences Jaume Almera, Barcelona, Spain. E-mail: schimmel@ictja.csic.es.

(C) 2012 Society of Exploration Geophysicists. All rights reserved.
} 
two main approaches: (1) They are generalized to represent seismic signals in a more compact way when signal trajectories can be modeled, or (2) they are adapted to be used in a local way. In surface seismics, the hyperbolic Radon transform is used in common-midpoint (CMP) gathers for multiple attenuation and noise removal (e.g., Yilmaz and Doherty, 2001). Trad et al. (2002, 2003) impose sparsity constraints to attain high-resolution Radon transforms for trace interpolation/regularization. Mann et al. (1999) (see also Jäger et al., 2001; Hertweck et al., 2007) propose the common-reflectionsurface (CRS) stack, as a local-second-order-approximation alternative, when CMP is not powerful enough or when its hyperbolic assumption is no longer valid. The LSST (Ottolini, 1983; Harlan et al., 1984; Bohlen et al., 2004; Shlivinski et al., 2005) is a local adaptation of the SST. This transform represents the data in the time-space-slowness domain, applying one low-aperture SST centered on each trace. As a result, the spatial resolution increases at the cost of "slowness resolution," with respect to the full-aperture SST. Note the term slowness resolution stands for "smallest interval measurable along the slowness axis in the LSST domain" and has little in common with the concept of slowness resolution of a velocity model in seismic migration or seismic tomography.

Widespread LSST applications are local-adaptive filters, e.g., the spatial-averaging filters on degree-of-polarization measures (Schimmel and Gallart, 2003, 2004), or the adaptive $f$ - $k$ filters of Duncan and Beresford (1994); and instantaneous slowness measures (Milkereit, 1987; van der Baan and Paul, 2000; Hu and Stoffa, 2009). Hence, the LSST is found in leading-edge algorithms, such as CRS, to estimate the first-order-approximation parameters or stereotomography (Billette and Lambaré, 1998; Billette et al., 2003; Lambaré, 2008) to measure local slope in the event picking.

A key element of these applications is the measurement of the local slope of the signal trajectory or instantaneous slowness. Fomel (2002, 2007a, b) and Schleicher et al. (2009) define slope-extraction techniques based on the derivative of the wavefield. In the LSST domain, this measurement is determined as a local maximum of the amplitude, envelope or coherence along the slowness axis.

Our goal is to estimate or to remove coherent signals by locally decomposing the seismic profiles in slowness using the LSST optimally. The main issues to solve in the design of a filter based on the LSST are the determination of the optimal aperture to locally distinguish the signals in the transformed domain and the choice of an appropriate synthesis operation of the filtered signals to the timespace domain. We use the good energy concentration of the LSST on seismic events to reduce the complexity of the synthesis operation. Regarding the first issue, the LSST window determines the aperture. The space-slowness location of a seismic signal in the LSST domain cannot be simultaneously known with an arbitrary high precision; the choice of the window entails a tradeoff between both. The spatial and the slowness resolution compromise set a minimum limit on the LSST window, whereas the coherency length (distance where signals remain similar) and the slope variations set a maximum. The coherency length generally decreases with increasing frequency due to subsurface structure complexities; in light of this dependency, Schimmel and Gallart (2007) adapt the window length with frequency.

The main task, when an LSST-based filter is employed, is to find a good tradeoff among key parameters, such as the slowness resolution, the interference and noise rejections, and the tracking capability of slowness-varying events. Conventionally, the optimum window is intuitively designed by visual inspection of some limited tests. To make this task easier and objective, we establish rigorous design criteria by analyzing the influence of the spatial-window bandwidth and the signal spectra on the slowness resolution of the LSST decomposition. As a result, we show that the optimum window can be designed beforehand, given the minimum global or local slowness resolution required to filter a seismic section.

This work is organized as follows. We first introduce the LSST and the adaptive filters based on this transform. We then focus on the slowness resolution estimation, stressing its main dependencies, and the synthesis of rigorous criteria on the LSST-window design. To conclude, we apply the new tools on synthetic and real data profiles and we discuss the main results.

\section{METHODOLOGY}

\section{Local-slant-stack transform}

The LSST of a seismic profile $u(t, x)$ with a weighting function $g(x)$, also called spatial-window, is

$$
v_{s}\left(\tau, x_{c}\right)=\int_{-\infty}^{+\infty} g\left(x-x_{c}\right) u\left(p_{s}\left(x-x_{c}\right)+\tau, x\right) \mathrm{d} x,
$$

where $\tau$ denotes the delay, $x_{c}$ the offset in the transformed domain, and $p_{s}$ the slowness, with $s$ its slowness index. Note that $g(x)$ is smooth, has unit area, and is positive around zero; and that $\tau$ and $x_{c}$ are continuous whereas $p_{s}$ is discrete.

In the discrete domain, the LSST of a seismic profile $u$ with a nonuniform separation between traces can be written as locally weighted sums of $L$ neighboring traces, $L$ being odd, along a set of signal wavefront trajectories of slowness $p_{s}$, with $s$ the slowness index

$$
v_{s, m}[n]=\sum_{i=-(L-1) / 2}^{(L-1) / 2} g_{m}[i] u_{m+i}\left(n T+\left(d_{m+i}-d_{m}\right) p_{s}\right),
$$

where each element $v_{s, m}[n]$ of the LSST decomposition is an estimation of the contribution of the signal with slowness $p_{s}$ to the sample $u_{m}[n]$, with $n$ and $m$ being the sample and trace indices, respectively. The time-space trajectory of the wavefront with a slowness $p_{s}$ is $t=n T+\left(d_{m+i}-d_{m}\right) p_{s}$, where $T$ is the sampling rate and $d_{m+i}-d_{m}$ the distance between the traces $u_{m}$ and $u_{m+i}$. The weight assigned to each trace, $g_{m}[i]$ may depend on the position of the central trace $u_{m}$, due to the usually nonuniform separation between traces.

Because the wavefront trajectories will not intersect the signal exactly at the time samples in equation 2, a polynomial interpolation is employed to find the values at arbitrary arrival times (Press et al., 2007). Additionally, note that the LSST assumes that seismic events vary slowly enough in slowness, amplitude, and shape to be locally approximated by plane waves.

\section{Filtering and synthesis}

Once the LSST, equation 2, has decomposed a seismic section in the time-space-slowness domain, we have to select and process the signals of interest and, finally, apply an inverse transform to synthesize a filtered seismic section in the time-space domain. Two opposite strategies can be followed in this inverse operation: synthesize the filtered profile estimating the signals to preserve, or estimate the 
signals to remove and subtract them from the original profile in the time-space domain.

Similar to the discrete inverse Radon transform, both strategies entail the solution of a large, sparse, linear system of equations. These operations are normally costly and can be unstable due to errors introduced by the use of finite-precision, real numbers. To minimize these problems, several inverse algorithms have been developed (see, e.g., Toft [1996] or Wilson and Guitton [2007] and the reference therein for the Radon transform, and Takiguchi [1995] or Shlivinski and Heyman [2009]) for the windowed version). However, these techniques do not consider that most of the energy of seismic signals is usually concentrated in a narrow slowness range. In this specific case, when the main signals are clearly separated, the inverse operation can be approximated by a sum of a small set of slowness components (Stoffa et al., 1981; Wenzel et al., 1982; van der Baan and Paul, 2000), or even by a single one (see, e.g., Schimmel and Gallart, 2003, 2004). Although full inverses are more accurate, a "lazy" inverse based on a single slowness component makes the inverse operation trivial, and it suffices for the applications of removing high-energy interfering waves and estimating low-energy waves, on which we focus.

We determine the instantaneous slowness of seismic waves in the LSST domain as the slowness value with a maximum degree of coherence (see, e.g., Milkereit, 1987; Duncan and Beresford, 1994). The most commonly used coherence estimators are the semblance and the normalized crosscorrelation (Neidell and Taner, 1971; Taner et al., 1979); but many other options based on eigendecompositions of the covariance matrix (Key and Smithson, 1990), phase stacks (Schimmel and Paulssen, 1997), or even 3D extensions like the coherence cube (Marfurt et al., 1998, 1999) are available. In this work, we opt for the phase-stack coherence estimator by Schimmel and Paulssen (1997) due to its noise robustness and amplitude unbiasedness. Using this criterion, the instantaneous slowness estimator can be written as

$$
q_{m}[n]=\arg \max \frac{1}{L}\left|\sum_{i=-(L-1) / 2}^{(L-1) / 2} \frac{u_{m+i, s}^{a}[n]}{\left|u_{m+i, s}^{a}[n]\right|}\right|
$$

being $u_{m+i, s}^{a}[n]=u_{m+i}^{a}\left(n T+\left(d_{m+i}-d_{m}\right) p_{s}\right)$, where $u_{m}^{a}(t)$ denotes the analytic representation of $u_{m}(t)$.

Finally, we can design LSST-based adaptive filters to isolate or to attenuate seismic signals, selecting the instantaneous slowness components measured by the previous estimators:

$$
y_{m}[n]=\left.v_{s, m}[n]\right|_{p_{s}=q_{m}[n]},
$$

where $q_{m}[n]$ denotes the instantaneous slowness and $v_{s, m}[n]$ is the seismic section decomposed in slowness.

\section{Slowness resolution}

Real seismic-signal trajectories do not have a constant slope. However, we can define the fundamental signal as locally coherent along a line of slope $q, u(t, x)=u(t-q x)$, thanks to the small slope variations of seismic signals. This approximation is valid while the slope variation is mild, as discussed in the Optimum window selection section. Introducing this constraint into the LSST integral, equation 1 , we obtain

$$
v_{s}\left(\tau, x_{c}\right)=\int_{-\infty}^{+\infty} g\left(x-x_{c}\right) u\left(\tau+p_{s}\left(x-x_{c}\right)-q x\right) \mathrm{d} x .
$$

Applying the change of variables $t_{1}=\tau+p_{s}\left(x-x_{c}\right)-q x$ and $d t_{1}=\left|q-p_{s}\right| d x$, we write

$$
v_{s}\left(\tau, x_{c}\right)=\int_{-\infty}^{+\infty} \frac{u\left(t_{1}\right)}{\left|q-p_{s}\right|} g\left(\frac{\tau-t_{1}-q x_{c}}{q-p_{s}}\right) \mathrm{d} t_{1},
$$

which can be expressed as a convolution over $t_{1}$

$$
v_{s}\left(\tau, x_{c}\right)=u \star \frac{1}{\left|q-p_{s}\right|} g\left(\frac{\tau-q x_{c}}{q-p_{s}}\right) .
$$

Equation 6, which is similar to a continuous wavelet transform (Daubechies, 1992; Mallat, 2009), can be seen as the inner product between $u(t)$ and a family of functions generated from $g(t)$ with translation and scaling operations. Despite the family of functions $g(t / a) /|a|$ being low pass and unit mean instead of having zero mean and unit energy as in wavelet theory, a fruitfull relationship can be established between the LSST and a generalization of the wavelet transform (Kaiser and Streater, 1992).

Defining the LSST family of functions $h_{s}(t)$ as

$$
h_{s}(t)=\frac{1}{\left|q-p_{s}\right|} g\left(\frac{t-q x_{c}}{q-p_{s}}\right) \text {, }
$$

for $p_{s} \neq q$, its Fourier transform is

$$
\hat{h}_{s}(\omega)=\hat{g}\left(\left(q-p_{s}\right) \omega\right) e^{-j q x_{c} \omega} .
$$

Note that $\omega$ denotes the temporal frequency and $k=\left(q-p_{s}\right) \omega$ is the spatial frequency. In the particular case of $p_{s}=q, h_{s}(t)$ is

$$
\lim _{p_{s} \rightarrow q} h_{s}(t)=\lim _{p_{s} \rightarrow q} \frac{1}{\left|q-p_{s}\right|} g\left(\frac{t-q x_{c}}{q-p_{s}}\right)=\delta\left(t-q x_{c}\right)
$$

in the sense of distributions, and its Fourier transform becomes

$$
\left.\hat{h}_{s}(\omega)\right|_{p_{s}=q}=e^{-j q x_{c} \omega}
$$

Hence, for $p_{s}=q, h_{s}(t)$ is an all-pass filter with a unit gain $\left.\hat{v}_{s}\left(\omega, x_{c}\right)\right|_{p_{s}=q}=\hat{u}(\omega) e^{-j q x_{c} \omega}$, or equivalently, $\left.v_{s}\left(t, x_{c}\right)\right|_{p_{s}=q}=$ $u\left(t-q x_{c}\right)$.

By applying the scaling property of the Fourier transform, $h(t)=g(t / a) \leftrightarrow \hat{h}(\omega)=|a| \hat{g}(a \omega)$, to the family of functions $\hat{h}_{s}(\omega)$, we notice that the $\hat{h}_{s}(\omega)$ bandwidth is inversely proportional to the $q-p_{s}$ factor and approaches infinity when $p_{s}$ approaches $q$, $\Delta \omega=\Delta k /\left|q-p_{s}\right|$, being $\Delta k$ the bandwidth of $\hat{g}(k)$. In other terms, when $p_{s} \neq q, h_{s}(t)$ becomes a low-pass filter whose bandwidth reduces as $\left|q-p_{s}\right|$ grows. Therefore, the high-frequency components are progressively attenuated as the $p_{s}$ slowness moves away from the $q$ slowness of the plane-wave $u(t-q x)$; until, if $\hat{u}(\omega)$ has a bandpass-like spectrum, the whole wave is almost completely removed. In conclusion, the characteristics of the spatial window and the wavefront spectrum fully determine the slowness resolution of the LSST and the coherent-signal attenuation. 
Several criteria to define the normalized bandwidth exist, e.g., the equivalent noise bandwidth, $\Delta k_{\mathrm{EN}}$, which represents the bandwidth that an ideal filter (infinite rejection in the stopband) would have to accumulate the same noise power. The minimum rejection to coherent signals or minimum stopband attenuation is defined in the frequency domain as the maximum peak level of the side lobes (stopband) with respect to the main lobe (passband). Measured in decibels, it reads

$$
A=10 \log _{10} \frac{\left|\hat{g}\left(k_{s l}\right)\right|^{2}}{|\hat{g}(0)|^{2}},
$$

where $k_{s l}$ is the peak spatial frequency of the side lobe with the highest amplitude. Table 1 summarizes the stopband attenuation and three normalized bandwidth criteria for several discrete windows. For a detailed analysis of these and other widely used windows, see, e.g., Harris (1978) and Oppenheim and Schafer (2009).

In the case of narrowband signals (bandwidth $\Delta \omega$ much lower than its central frequency $\omega_{0}$ ), the response of the LSST at the central frequency $\omega_{0}$ is

$$
\hat{h}_{s}\left(\omega_{0}\right)=\hat{g}\left(\left(q-p_{s}\right) \omega_{0}\right) e^{-j q x_{c} \omega_{0}},
$$

hence the slowness resolution at $\omega_{0}$ is

$$
\Delta p=\Delta k / \omega_{0},
$$

where $\Delta k$ is the spatial-frequency bandwidth of $\hat{g}(k)$. The location of the side lobes in slowness depends on the inverse of $\omega_{0}$, $p_{s l}=q \pm k_{s l} / \omega_{0}$, whereas the minimum slowness rejection is independent of $\omega_{0}$ and equals the stopband attenuation.

For example, the slowness resolution of an LSST that uses a rectangular window of length $L$ in the narrowband approximation is

$$
\Delta p=\frac{\Delta k}{\omega_{0}}=\frac{2 \pi \Delta k_{\mathrm{EN}}}{L \omega_{0}}=\frac{2 \pi}{L \omega_{0}}=\frac{1}{L f_{0}},
$$

where $f_{0}$ is the central frequency of the signal in $\mathrm{Hz}$, and $\Delta k$ is the spatial-window bandwidth in $1 / \mathrm{m}$. In this case, we define $\Delta k$ using the equivalent noise bandwidth $\Delta k_{\mathrm{EN}}$ criterion, Table 1 . The minimum slowness rejection is $A=-13 \mathrm{~dB}$.

\section{Optimum window selection}

The main features to set in an LSST are the slowness resolution, the stopband attenuation, and the wavefront-tracking capability. As shown in the slowness resolution section, given a wave with a pass- band spectrum, all these features are determined by the spatial window.

\section{Window length}

From the viewpoint of maximizing the wavefront-tracking capability, the optimal window is the shortest one that fulfills the minimum slowness resolution to attain the maximum resolution in the offset axis. However, note that as the window gets shorter, the sensitivity to noise increases.

In narrowband signals, the relationship between slowness resolution and window bandwidth, equation 14, determines the minimum length of the LSST window to attain a minimum slowness resolution, $\Delta p_{\min } \geq \Delta k / 2 \pi f_{0}$. As the window bandwidth is $\Delta k=$ $2 \pi \Delta k_{\mathrm{N}} / L$, being $\Delta k_{\mathrm{N}}$ a normalized bandwidth, the window length must be

$$
L \geq \frac{\Delta k_{\mathrm{N}}}{\Delta p_{\min } f_{0}}
$$

to fulfill the minimum slowness resolution. The most rigorous definition of the $\Delta k_{\mathrm{N}}$ bandwidth establishes the stopband attenuation at $\Delta p_{\text {min }}$ from the slowness of the signal to estimate. When defined, we approximate this bandwidth by the bandwidth between zeros.

In wideband signals, i.e., when $\Delta \omega \gg \omega_{0}$, the lowest frequency components determine the window length due to the inversely proportional relationship between frequency and window length,

$$
L \geq \frac{\Delta k_{\mathrm{N}}}{\Delta p_{\min } f_{\min }},
$$

where $f_{\min }$ denotes the minimum meaningful frequency.

\section{Window function}

The main criterion to choose the window function is the desired minimum rejection. When we have to remove a large amplitude wave that masks a weaker one, beside having enough resolution in slowness to distinguish them, the stopband attenuation should be much greater than the ratio of their amplitudes, otherwise, the weaker wave can be completely masked or distorted by the side lobes of the stronger one. Note that attenuations that are too high must be avoided due to the inherent increase in bandwidth (see, e.g., Table 1) which implies longer windows (equation 16) and consequently a decreased wavefront-tracking capability.

Table 1. Normalized bandwidths in bins (frequency unit of the discrete Fourier transform) and rejection of several windows. $\Delta k_{-3 \mathrm{~dB}}$ is the $-\mathbf{3} \mathrm{dB}$ bandwidth, $\Delta \boldsymbol{k}_{\mathrm{EN}}$ the equivalent noise bandwidth, $\Delta \boldsymbol{k}_{z}$ the bandwidth between zeros and $A$ the stopband attenuation.

\begin{tabular}{lcccc}
\hline Name & $g[n] n=-N / 2 \ldots N / 2$ & $\Delta k_{-3 \mathrm{~dB}}(\mathrm{bins})$ & $\Delta k_{\mathrm{EN}}(\mathrm{bins})$ & $\Delta k_{z}(\mathrm{bins})$ \\
\hline Rectangular & 1 & 0.89 & 1.00 & 2 \\
(Co)sine & $\cos \left(\frac{\pi n}{N}\right)$ & 1.20 & 1.23 & -13 \\
Triangle & $1-\left|\frac{2 n}{N}\right|$ & 1.28 & 1.33 & -23 \\
Hamming & $0.54+0.46 \cos \left(\frac{2 \pi n}{N}\right)$ & 1.30 & 1.36 & -27 \\
Gaussian & $e^{-18(n / N)^{2}}$ & 1.55 & 1.64 & -43 \\
\hline
\end{tabular}


Optimum window for events of moderately varying slowness

When an LSST-based filter is used to remove coherent signals, it is important to remember the assumption of mild slowness variations along the offset implicitly made with the LSST. As long as

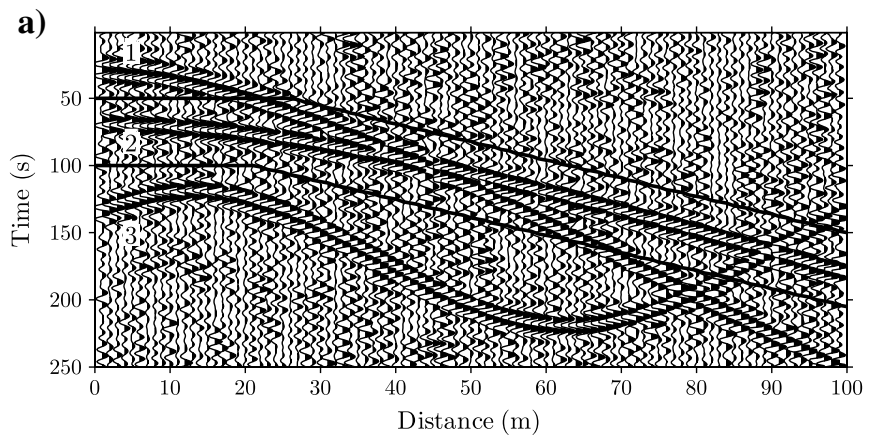

this key assumption is valid for the window length used, the results will be close to the expected ones.

The amplitude envelope of a wave changes with the offset much more slowly than does its phase; as a result, the error in

Figure 1. Synthetic seismic data set composed of three seismic waves of variable slowness and Gaussian noise. (a) Original section with the work area delimited by black lines. (b) LSST-filtered section.
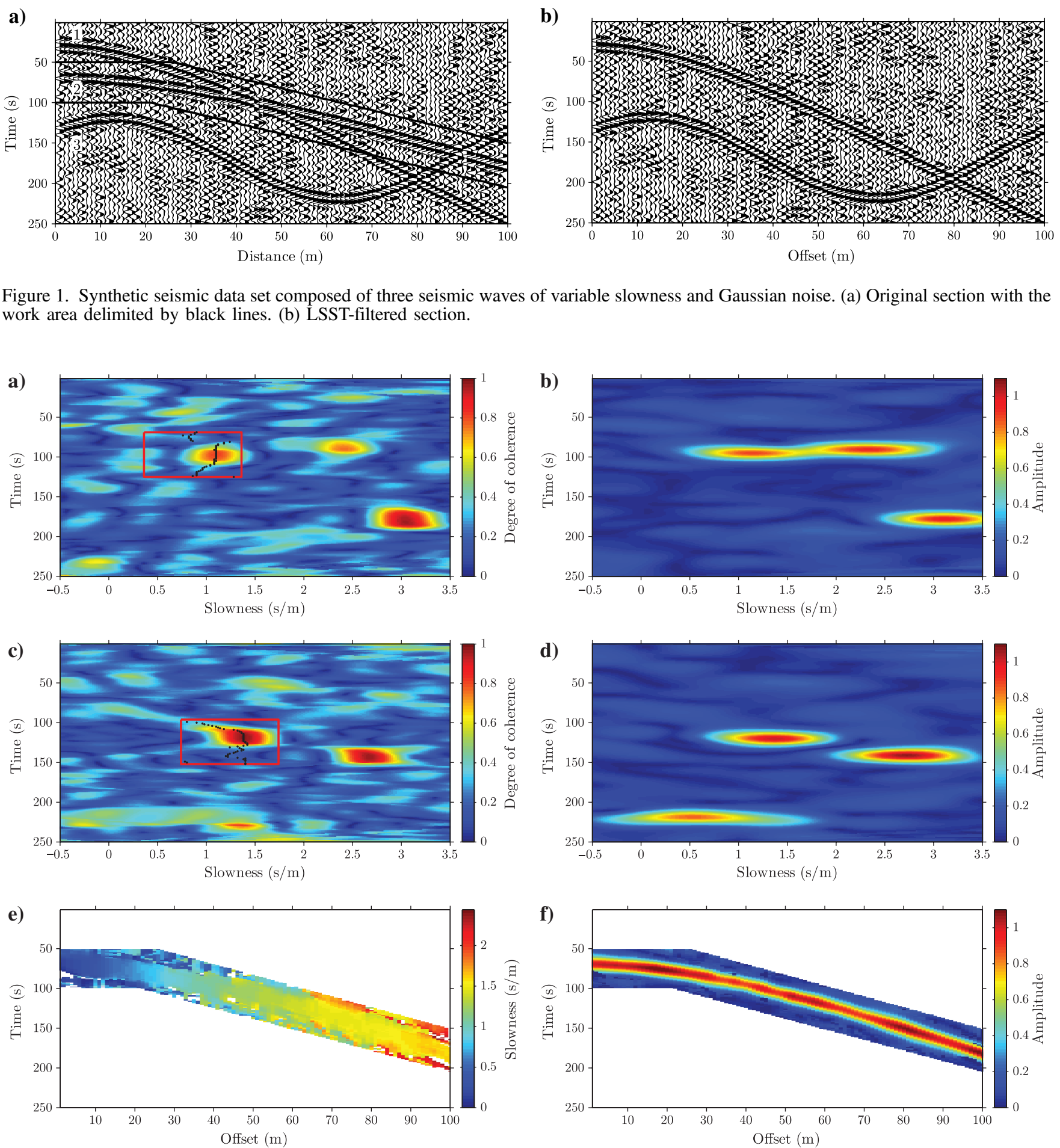

Figure 2. Filtering algorithm details. Degree-of-coherence sections (a) at $40 \mathrm{~m}$ and (c) at $60 \mathrm{~m}$, where black dots indicate slowness maxima and solid lines the working domain. LSST-modulus sections (b) at $40 \mathrm{~m}$ and (d) at $60 \mathrm{~m}$. (e) Estimated instantaneous slowness. (f) Modulus of the estimated signal. 
the estimation of signals of moderately varying slowness is mostly concentrated in their phases. When the phase shifts are in the same direction at opposite sides of the window with respect to its center, such as around the zero offset in a reflection event, the instantaneous slowness measure remains low biased, but the phase of the signal estimation does not. On the contrary, when the shifts are in opposite directions, the signal estimation has a low-phase bias whereas the slowness measure does not.

To minimize this effect, the maximum weight has to be given to the traces close to the window center; as a consequence, the most appropriate function is the rectangular window, thanks to its high processing gain. When the slowness variations are severe and this minimization is insufficient, then one can use alternative approaches based on second-order approximations, such as CRS-based filters.

\section{EXAMPLES AND DISCUSSION}

To evaluate the design rules for an optimum LSST-window selection shown in the previous section, we apply an LSST-based filter to attenuate high-energy waves using synthetic data first and then real data. To clearly illustrate the compromises to solve, we use a unique spatial window along the synthetic profile; however, we remove this constraint in real data to unlock the full potential of the LSST.

\section{Three seismic waves of variable slowness}

The synthetic seismic profile shown in Figure 1a contains three unit-amplitude slowness-varying events contaminated by Gaussian additive noise in which the sampling rate is normalized to $1 \mathrm{sample} / \mathrm{s}$ and the trace separation to $1 \mathrm{~m}$. The events have a

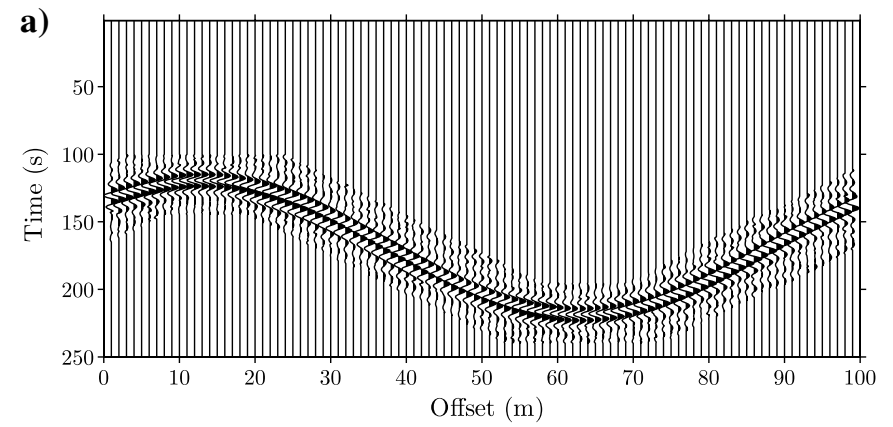

modulated Gaussian waveform with a normalized central frequency of $f_{0}=1 / 9 \mathrm{~Hz}$ ( 9 samples/cycle), and the noise has a variance of 0.05 and the same amplitude spectrum as the signals. The slowness of signals 1 and 2 (labeled in Figure 1) increases progressively from zero at the origin to 3 and $1 \mathrm{~s} / \mathrm{m}$, respectively, at $100 \mathrm{~m}$. The slowness range of signal 3 includes the range of signals 1 and 2 . Note that the first two signals intersect in the central part of the section, and that their slowness ranges overlap. Although signal 2 is interfering in slowness or time with the other signals, the LSST-based filter successfully removes it, see Figure $1 \mathrm{~b}$. These results cannot be obtained with a filter in the $\tau$ - $p$ domain because of the high slowness variations along the offset, nor with a pie-slice $f-k$ filter, due to the overlap of the slowness ranges.

The signals overlap with the minimal slowness difference of $1.1 \mathrm{~s} / \mathrm{m}$ just before the intersection between signals 1 and 2, at approximately $30 \mathrm{~m}$. This implies a $\Delta p_{\min } \geq 2.2 \mathrm{~s} / \mathrm{m}$, which, according to equation 16, leads to an LSST-window length of $L \geq 4.09 \Delta k_{\mathrm{N}}$ m to have enough slowness resolution to distinguish both signals. As shown in the previous section, the window bandwidth is determined by the desired minimum rejection and the employed definition criterion. Assuming that we require a distortion lower than $10 \%$ on signal amplitude, a sine window with a stopband attenuation of $-23 \mathrm{~dB}$ is admissible. Consequently, approximating the stopband bandwidth by the bandwidth between zeros, $\Delta k_{\mathrm{N}} \simeq \Delta k_{z}=3$, the minimum window length is $13 \mathrm{~m}$.

Figure $2 \mathrm{~b}$ and $2 \mathrm{~d}$ show time-slowness panels of the modulus of the LSST at 40 and $60 \mathrm{~m}$. In both, the three signals are clearly separated and, in particular, the slowness resolution is sufficient to distinguish signal 1 from signal 2 . The number of slowness

Figure 3. Attenuation of the third wave of Figure 1. (a) Estimated signal. (b) Filtered record section.
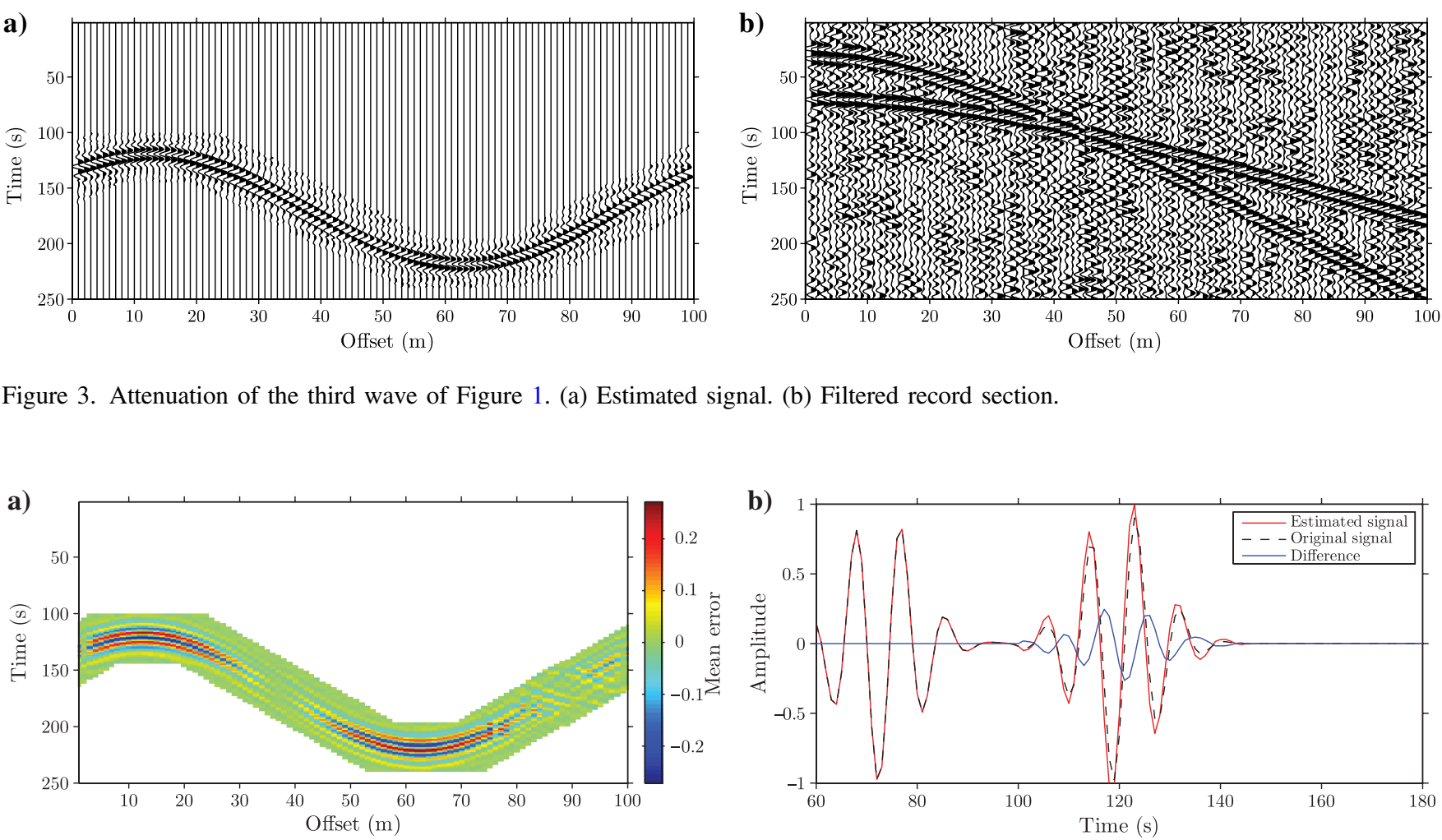

Figure 4. Mean-amplitude error in the estimation of the third signal of Figure 3 with respect to the original but without noise. (a) Full section. (b) Comparison between the original noiseless signal and the estimated one at the trace located at $12 \mathrm{~m}$. 
components depends on the highest resolution achieved so that the introduced quantization error is negligible. A Lagrange polynomial interpolation of order five is used to interpolate all the required points at each trace.

The instantaneous slowness is measured using a phase-stack coherence estimator with a rectangular window of $17 \mathrm{~m}$, equation 3 . Figure $2 \mathrm{a}$ and 2c display time-slowness coherence panels at 40 and $60 \mathrm{~m}$, together with the slowness maxima (black points) in the working domain (solid lines). The window length employed in the degree-of-coherence estimator is usually longer than the one used in the LSST, thanks to the low time variation of the instantaneous slowness of the signal. The resulting slowness resolution increase allows a better measure of the instantaneous slowness along offset than the local maxima of the LSST modulus, see Figure 2e, and as a consequence, a more accurate location of the signal in the LSST domain, see Figure 2f. For this reason, we employ the slowness estimation in the synthesis operation, equation 4.

Finally, we illustrate the limitation of the LSST-based filters on the estimation of events of moderately varying slowness. The aim is now to attenuate signal 3 of Figure 1a that has a maximum slowness variation along the offset axis of $0.2 \mathrm{~s} / \mathrm{m}^{2}$, twice the maximum of signal 2 and six times the maximum in the overlapping zone between signals 1 and 2. As the minimum slowness resolution is not constrained, we use a rectangular window of $7 \mathrm{~m}$ for the LSST and of $11 \mathrm{~m}$ for the instantaneous slowness estimation.

Although the phase bias in the estimated and the filtered sections shown in Figure 3 is too small to distinguish it, this error can be clearly seen in Figure 4 where we show the mean error of different noise realizations between the original and the estimated signals. As discussed in the optimum window selection section, the phase bias is higher at slowness variation maxima. In this example, the maximum error is approximately a quarter of the amplitude of the original signal.

\section{Real data example: Attenuation of high-energy waves}

The profile shown in Figure 5 is a zoom of a vertical-component ocean bottom seismometer (OBS) record section used in the MARCONI project (OBS 16, profile 13) to study the continental margin at the transition of the Eurasian and Iberian plates in the Bay of Biscay (Ruiz, 2007). The main targets are reflections and refractions from the lower crust that are obscured at certain distances by the arrival of strong direct waves (between sources and OBS) and water-bottom multiples. The goal is to attenuate these high-energy signals, which we refer to as water waves, to unravel the hidden weak signals.

The highly coherent signals with high slope (low apparent velocity) in Figure 5 are the water waves generated by previous air-gun shots. Waves reflected and refracted from discontinuities in the lower crust and upper-most mantle and their multiples are seen as horizontal arrivals due to the reduction velocity of $8 \mathrm{~km} / \mathrm{s}$ applied to the time axis. An LSST-based filter is applied to this data set to attenuate the water waves while keeping the distortion of the other waves, and particularly of the refracted waves, at the minimum level.
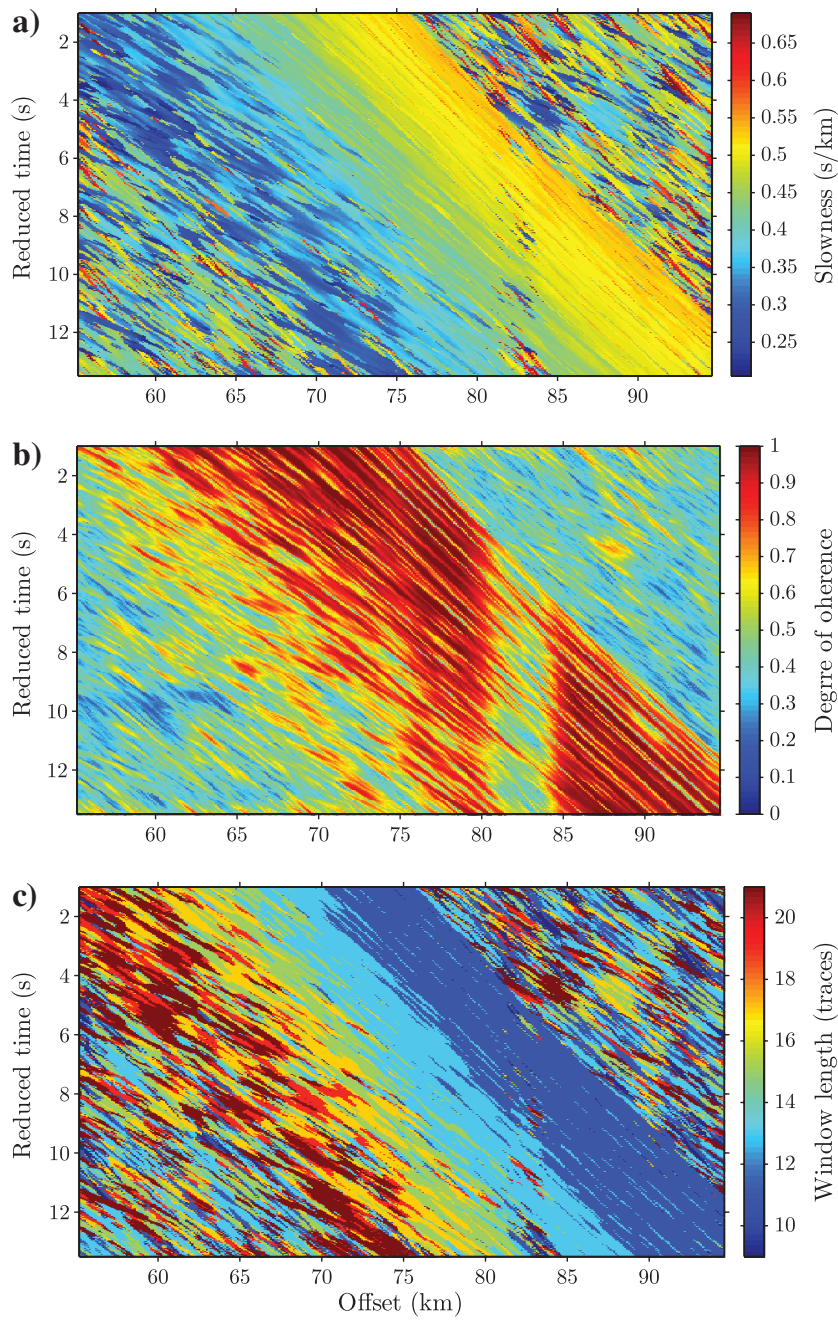

Figure 6. Filtering algorithm details. (a) Slowness measures of the water wave and (b) their degree of coherence using the phase-stack estimator. (c) Optimum length of the LSST window in number of traces $(1 \mathrm{~km} \simeq 9$ traces $)$. 
The instantaneous slowness of the water waves and the refracted waves clearly varies along the profile. Their slowness ranges extend from 0.25 to $0.55 \mathrm{~s} / \mathrm{km}$ and from -0.14 to $0.07 \mathrm{~s} / \mathrm{km}$, respectively. The minimum difference along the whole profile between these slowness bands is $0.18 \mathrm{~s} / \mathrm{km}$, which implies a global $\Delta p_{\min } \geq$ $0.36 \mathrm{~s} / \mathrm{km}$.

The high-amplitude difference between the waves of interest and the interfering signals imposes a high stopband attenuation constraint to keep refracted events undistorted and to limit artifacts. To fulfill this constraint, we choose a Hamming window due to its high stopband attenuation $(-43 \mathrm{~dB})$ for its bandwidth; which leads to a window length of $L \geq 0.8 \mathrm{~s} / \Delta p_{\min }=2.2 \mathrm{~km}$, equation 16 , considering a minimum meaningful frequency of $5 \mathrm{~Hz}$ and approximating the stopband bandwidth by $\Delta k_{\mathrm{N}} \simeq$ $\Delta k_{z}=4$. Furthermore, we select a phase-stack coherence estimator with a Hamming window of 31 traces $(3.5 \mathrm{~km})$ to measure the instantaneous slowness of the water waves and their degree of coherence, see Figure 6.

The above global criterion provides good enough results; however, we can obtain better results with a negligible computational cost increase by applying the minimum slowness resolution criterion locally. In this example, we locally increase the $\Delta p_{\min }$ constraint, replacing the minimum slowness of the water waves by their instantaneous estimation. Figure $6 \mathrm{c}$ shows the minimum window length required after being rounded upward to the nearest odd integer. The filtered profile is obtained by first measuring the single LSST-instantaneous-slowness component of the water waves and then subtracting the result from the original profile.
As Figure 7 shows, the LSST-based filter successfully removes the water waves; this enables the analysis of refracted waves at offsets where they were totally masked. Along the first seconds of the water waves at a given trace, where the signal is strong and the lateral amplitude variations small, the disturbing waves are totally imperceptible. However, as time increases and amplitude decreases, lateral amplitude variations grow and, as a consequence, a small residual of the water waves can be noticed.

In fact, these variations introduce a constraint on the LSST design. While the lateral amplitude variations increase, the spatialwindow length has to be reduced to improve the amplitude-tracking capabilities of the LSST. We have used a rigorous bandwidth definition to keep the artifacts at a minimum level, Figure 7. But, less rigorous definitions, that lead to shorter windows and thus better amplitude-tracking capabilities, could also be applied.

Figure 8 depicts the data in the $f-k$ domain together with the results obtained using a pie-slice $f-k$ filter with a stopband that ranges from one to three samples/trace $(0.22$ to $0.67 \mathrm{~s} / \mathrm{km})$, and the LSSTbased filter. Although the water waves seem to be successfully removed in both cases, the results are totally different. The $f-k$ filter removes an important sector of the transformed domain, Figure 8b, whereas the LSST filter keeps this sector with a far lower distortion, Figure $8 \mathrm{c}$. Although the noise characteristics of the original and the LSST-filtered profiles are similar, compare Figures 5 and 7, most of the high spatial-frequency components are removed in the $f-k$ filtered profile, Figure 9. Due to this spatial averaging, the strongest refracted waves are better perceived, but it is far more difficult to distinguish smaller signals from the noise.
Figure 7. (a) Estimation of the wave to remove and (b) the LSST-filtered profile after the subtraction.
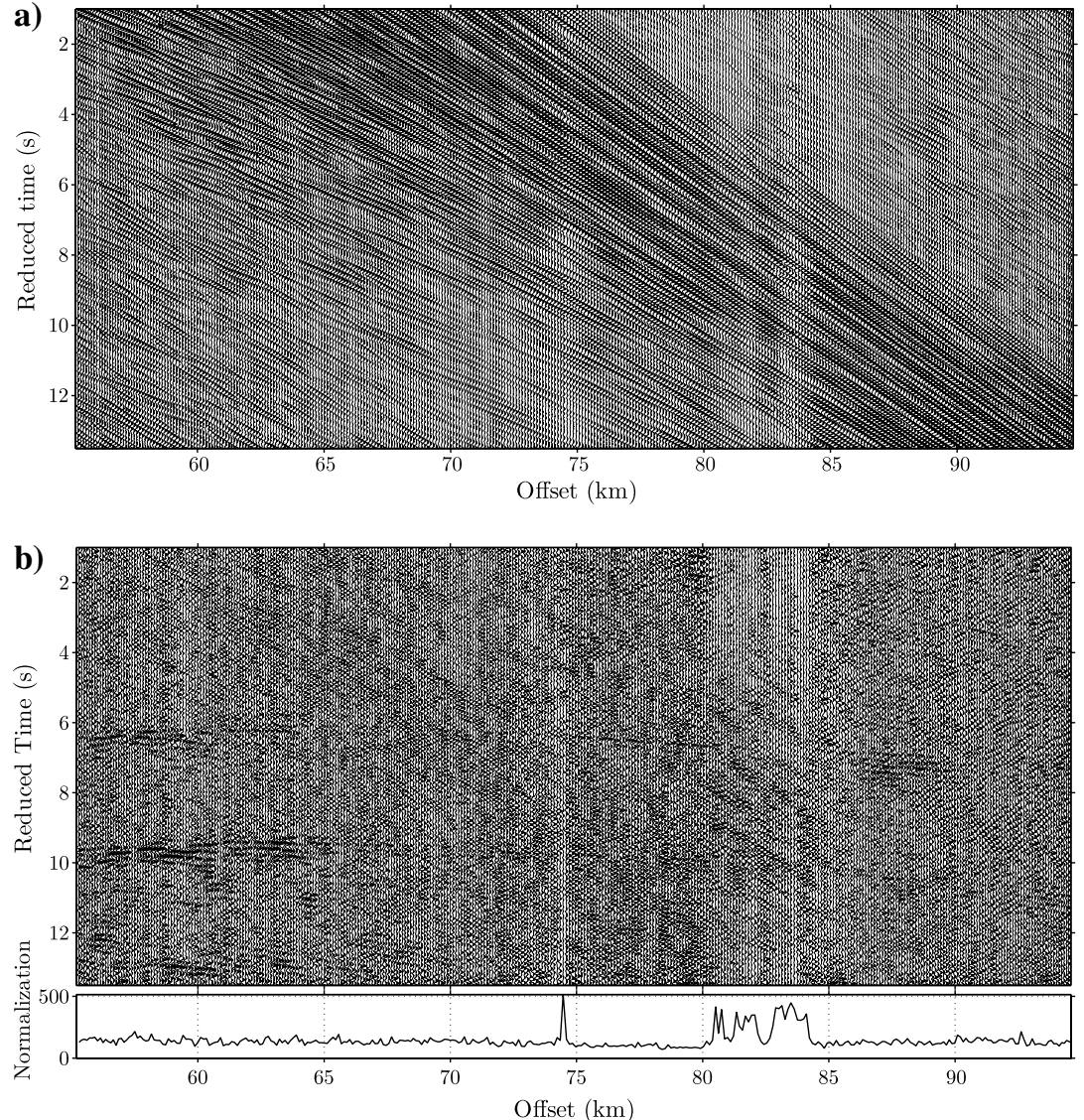

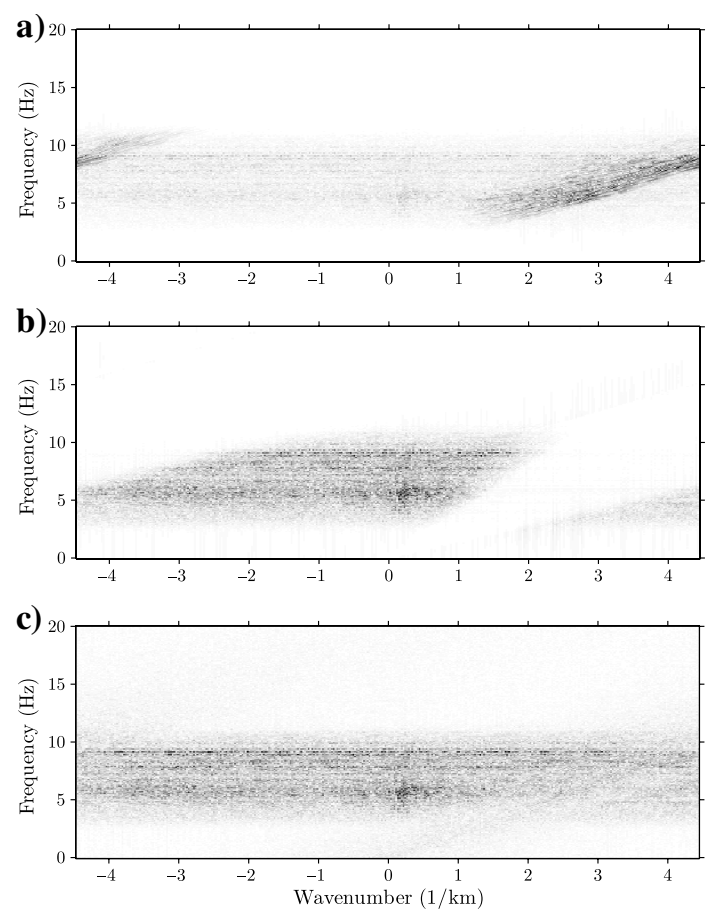

Figure 8. Representation in the $f-k$ domain of (a) the original, (b) the $f-k$ filtered, and (c) the LSST-filtered profiles. It clearly illustrates the minimum distorsion of the LSST method with respect to the $f$ - $k$ filter.
It is well known that coherent artifacts may be generated when too sharp $f-k$ filters are used. This can lead to misinterpretations in a complicated processing flow where other tools are applied to remove incoherent waves or where certain attributes are extracted. Thanks to the great flexibility that the local adaptation of the LSST-based filters provides, we can reduce the risk of generating artifacts using softer and local adaptive filters. Note that the attenuation of the incoherent noise that further obscures the reflected and the refracted signals has not been the objective of this contribution. The objective has focused on estimating and subtracting the coherent water waves with a minimum distortion; other processing tools can now be applied to attenuate the incoherent noise, see, e.g., Schimmel and Gallart (2007) for a possible approach to further clean this profile.

\section{CONCLUSIONS}

Plane-wave-decomposition techniques, such as pie-slice $f$ - $k$ filters or filters in the $\tau$ - $p$ domain, cannot be successfully applied to filter signals with slowness variations due to their lack of spatial resolution. This limitation is surpassed by employing filters based on the LSST. The most critical decision to take in the design of the LSST is the selection of the length and the shape of the spatial window. The challenge is to achieve a compromise between the slowness and the spatial resolutions. To solve this problem in a rigorous way, we have estimated the slowness resolution in the LSST domain as a function of the window bandwidth and signal spectrum. This result has allowed us to select the window that achieves the minimum slowness resolution required to estimate the signal of interest, minimizing the interference caused by other signals. Longer windows can only be used when the slowness and the amplitude variations of the signal-wavefront trajectories are small. However, if these variations are strong, this window length guarantees that the signals of interest are estimated with the minimum number of traces. This permits us to increase the trackability of variable signals to a quantified optimum resolution. The criteria of minimum slowness resolution in the LSST domain can be used to select the optimum spatial-window automatically, either globally or in a data-adaptive fashion, depending on instantaneous slowness measures. But, these criteria can also be useful in key elements of modern tools, such as the estimation of the approximation parameters in CRS or the local slope in stereotomography.

\section{ACKNOWLEDGMENTS}

The authors thank the reviewers, the editors, and Laurent Duval for their useful comments that greatly improve the quality of the manuscript. This publication originates in the Ph.D. thesis of Sergi Ventosa at UTM-CSIC. Part of this research was possible thanks to the project Nearest (CE-037110). The work of Carine Simon is supported by the Ramon y Cajal program, and the work of Martin Schimmel through the

Figure 9. The $f-k$ removed signals (a) and the filtered profile (b). 
Consolider-Ingenio 2010 program Nr. CSD2006-00041 (Topo-Iberia) and the PN Nr. CGL2009-09727 (Rifsis).

\section{REFERENCES}

Bekara, M., and M. van der Baan, 2009, Random and coherent noise attenuation by empirical mode decomposition: Geophysics, 74, no. 5, V89-V98, doi: 10.1190/1.3157244.

Billette, F., S. L. Bégat, P. Podvin, and G. Lambaré, 2003, Practical aspects and applications of 2d stereotomography: Geophysics, 68, 1008-1021, doi: $10.1190 / 1.1581072$.

Billette, F., and G. Lambaré, 1998, Velocity macro-model estimation from seismic reflection data by stereotomography: Geophysical Journal International, 135, 671-690, doi: 10.1046/j.1365-246X.1998 $.00632 . x$.

Bohlen, T., S. Kugler, G. Klein, and F. Theilen, 2004, 1.5D inversion of lateral variation of scholte-wave dispersion: Geophysics, 69, 330-344, doi: 10.1190/1.1707052.

Daubechies, I., 1992, Ten lectures on wavelets: SIAM.

Deans, S. R., 1993, The radon transform and some of its applications: Krieger Publishing Company.

Dev, A., and G. A. McMechan, 2009, Spatial antialias filtering in the slowness-frequency domain: Geophysics, 74, no. 2, V35-V42, doi: 10.1190/1 .3052115 .

Duncan, G., and G. Beresford, 1994, Slowness adaptive $f-k$ filtering of prestack seismic data: Geophysics, 59, 140-147, doi: 10.1190/1.1443525.

Durrani, T., and D. Bisset, 1984, The Radon transform and its properties: Geophysics, 49, 1180-1187, doi: 10.1190/1.1441747.

Embree, P., J. P. Burg, and M. M. Backus, 1963, Wide-band velocity filtering — The pie-slice process: Geophysics, 28, 948-974, doi: 10 $.1190 / 1.1439310$.

Fomel, S., 2002, Applications of plane-wave destruction filters: Geophysics, 67, 1946-1960, doi: 10.1190/1.1527095.

Fomel, S., 2007a, Shaping regularization in geophysical-estimation problems: Geophysics, 72, no. 2, R29-R36, doi: 10.1190/1.2433716.

Fomel, S., 2007b, Velocity-independent time-domain seismic imaging using local event slopes: Geophysics, 72, no. 3, S139-S147, doi: 10 $1190 / 1.2714047$

Forbriger, T., 2003, Inversion of shallow-seismic wavefields: I. Wavefield transformation: Geophysical Journal International, 153, 719-734, doi: 10.1046/j.1365-246X.2003.01929.x.

Guitton, A., 2005, Multiple attenuation in complex geology with a patternbased approach: Geophysics, 70, no. 4, V97-V107, doi: 10.1190/ 1.1997369

Harlan, W. S., J. F. Claerbout, and F. Rocca, 1984, Signal/noise separation and velocity estimation: Geophysics, 49, 1869-1880, doi: 10.1190/ 1.1441600 .

Harris, F. J., 1978, On the use of windows for harmonic analysis with the discrete Fourier transform: Proceedings of the IEEE, 66, 51-83, doi: 10 .1109/PROC.1978.10837.

Hertweck, T., J. Schleicher, and J. Mann, 2007, Data stacking beyond cmp: The Leading Edge, 26, 818-827, doi: 10.1190/1.2756859.

$\mathrm{Hu}, \mathrm{C}$. , and P. L. Stoffa, 2009, Slowness-driven gaussian-beam prestack depth migration for low-fold seismic data: Geophysics, 74, no. 6, WCA35-WCA45, doi: 10.1190/1.3250268.

Jäger, R., J. Mann, G. Höcht, and P. Hubral, 2001, Common-reflectionsurface stack: Image and attributes: Geophysics, 66, 97-109, doi: 10 $.1190 / 1.1444927$.

Kaiser, G. A., and R. F. Streater, 1992, Windowed Radon transforms, analytic signals and the wave equation, in C. K. Chui, ed., Wavelets: A tutorial in theory and applications: Academic Press Geology Series, 39, 9-441.

Key, S. C., and S. B. Smithson, 1990, New approach to seismic-reflection event detection and velocity determination: Geophysics, 55, 1057-1069, doi: 10.1190/1.1442918.

Lambaré, G., 2008, Stereotomography: Geophysics, 73, no. 5, VE25-VE34, doi: 10.1190/1.2952039.

Mallat, S., 2009, A wavelet tour of signal processing, 3rd ed.: The sparse way: Academic Press.

Mann, J., R. Jöger, T. Müller, G. Höcht, and P. Hubral, 1999, Commonreflection-surface stack - A real data example: Journal of Applied Geophysics, 42, 301-318, doi: 10.1016/S0926-9851(99)00042-7.

Marfurt, K. J., R. L. Kirlinz, S. L. Farmer, and M. S. Bahorich, 1998, 3-D seismic attributes using a semblance-based coherency algorithm: Geophysics, 63, 1150-1165, doi: 10.1190/1.1444415.

Marfurt, K. J., V. Sudhakerz, A. Gersztenkorn, K. D. Crawford, and S. E. Nissen, 1999, Coherency calculations in the presence of structural dip: Geophysics, 64, 104-111, doi: 10.1190/1.1444508.
Milkereit, B., 1987, Decomposition and inversion of seismic data - An instantaneous slowness approach: Geophysical Prospecting, 35, 875894, doi: $10.1111 /$ gpr.1987.35.issue-8.

Neidell, N. S., and M. T. Taner, 1971, Semblance and other coherency measures for multichannel data: Geophysics, 36, 482-497, doi: 10.1190/ 1.1440186.

Oppenheim, A. V., and R. W. Schafer, 2009, Discrete-time signal processing, 3rd edition: Prentice Hall.

Ottolini, R., 1983, Signal/noise separation in dip space: Stanford Exploration Project-37, 143-149.

Press, W. H., S. A. Teukolsky, W. T. Vetterling, and B. P. Flannery, 2007, Numerical recipes: The art of scientific computing, 3rd ed.: Cambridge University Press.

Ruiz, M., 2007, Caracterització estructural i sismotectònica de la litosfera en el domini pirenaico-cantàbric a partir de mètodes de sísmica activa i passiva: Ph.D. thesis Universitat de Barcelona.

Schimmel, M., and J. Gallart, 2003, The use of instantaneous polarization attributes for seismic signal detection and image enhancement: Geophysical Journal International, 155, 653-668, doi: 10.1046/j.1365-246X 2003.02077.x.

Schimmel, M., and J. Gallart, 2004, Degree of polarization filter for frequency-dependent signal enhancement through noise suppression: Bulletin of the Seismological Society of America, 94, 1016-1035, doi: 10 $1785 / 0120030178$.

Schimmel, M., and J. Gallart, 2007, Frequency-dependent phase coherence for noise suppression in seismic array data: Journal of Geophysical Research, 112, B04303, doi: 10.1029/2006JB004680.

Schimmel, M., and H. Paulssen, 1997, Noise reduction and detection of weak, coherent signals through phase-weighted stacks: Geophysical Journal International, 130, 497-505, doi: 10.1111/gji.1997.130 .issue-2.

Schleicher, J., J. C. Costa, L. T. Santos, A. Novais, and M. Tygel, 2009, On the estimation of local slopes: Geophysics, 74, no. 4, P25-P33, doi: 10 $.1190 / 1.3119563$

Shlivinski, A., and E. Heyman, 2009, Windowed Radon transform frames: Applied and Computational Harmonic Analysis, 26, 322-343, doi: 10 .1016/j.acha.2008.07.003.

Shlivinski, A., E. Heyman, and A. Boag, 2005, A pulsed beam summation formulation for short pulse radiation based on windowed Radon transform (WRT) frames: IEEE Transactions on Antennas and Propagation, 53, 3030-3048, doi: 10.1109/TAP.2005.854550.

Stoffa, P. L., P. Buhl, J. B. Diebold, and F. Wenzel, 1981, Direct mapping of seismic data to the domain of intercept time and ray parameter - A plane-wave decomposition: Geophysics, 46, 255-267, doi: 10.1190/ 1.1441197.

Takiguchi, T., 1995, On invertibility of the windowed Radon transform: Journal of Mathematical Sciences, The University of Tokyo, 2, 621-636.

Taner, M. T., F. Koehler, and R. E. Sheriff, 1979, Complex seismic trace analysis: Geophysics, 44, 1041-1063, doi: 10.1190/1.1440994.

Toft, P., 1996, The Radon transform: Theory and implementation: Ph.D. thesis, Technical University of Denmark.

Trad, D., T. Ulrych, and M. Sacchi, 2003, Latest views of the sparse Radon transform: Geophysics, 68, 386-399, doi: 10.1190/1.1543224.

Trad, D., T. J. Ulrych, and M. Sacchi, 2002, Accurate interpolation with high-resolution time-variant Radon transforms: Geophysics, 67, 644656, doi: $10.1190 / 1.1468626$.

Turner, G., 1990, Aliasing in the tau-p transform and the removal of spatially aliased coherent noise: Geophysics, 55, 1496-1503, doi: 10.1190/ 1.1442797.

van der Baan, M., and A. Paul, 2000, Recognition and reconstruction of coherent energy with application to deep seismic reflection data: Geophysics, 65, 656-667, doi: 10.1190/1.1444763.

Vrabie, V. D., N. L. Bihan, and J. I. Mars, 2006, Multicomponent wave separation using HOSVD/unimodal-ICA subspace method: Geophysics, 71, no. 5, V133-V143, doi: 10.1190/1.2335387.

Vrabie, V. D., J. I. Mars, and J. L. Lacoume, 2004, Modified singular value decomposition by means of independent component analysis: Signal Processing Magazine, 84, 645-652, doi: 10.1016/j.sigpro.2003.12.007.

Wenzel, F., P. Stoffa, and P. Buhl, 1982, Seismic modeling in the domain of intercept time and ray parameter: IEEE Transactions on Acoustics, Speech, and Signal Processing, 30, 406-423, doi: 10.1109/TASSP .1982 .1163906 .

Wilson, C., and A. Guitton, 2007, Teleseismic wavefield interpolation and signal extraction using high-resolution linear radon transforms: Geophysical Journal International, 168, 171-181, doi: 10.1111/gji.2007.168 issue-1.

Yilmaz, O., and S. M. Doherty, 2001, Seismic data analysis: Processing, inversion, and interpretation of seismic data: SEG. 